\title{
Bu open Adherence management for patients with cancer taking capecitabine: a prospective two-arm cohort study
}

\author{
Linda Krolop, ${ }^{1}$ Yon-Dschun Ko, ${ }^{2}$ Peter Florian Schwindt, ${ }^{3}$ Claudia Schumacher, ${ }^{4}$ \\ Rolf Fimmers, ${ }^{5}$ Ulrich Jaehde ${ }^{1}$
}

To cite: Krolop L, Ko Y-D, Schwindt PF, et al. Adherence management for patients with cancer taking capecitabine: a prospective two-arm cohort study. BMJ Open 2013;3: e003139. doi:10.1136/ bmjopen-2013-003139

- Prepublication history and additional material for this paper is available online. To view these files please visit the journal online (http://dx.doi.org/10.1136/ bmjopen-2013-003139).

Received 29 April 2013 Revised 28 May 2013 Accepted 17 June 2013
${ }^{1}$ Institute of Pharmacy, Clinical Pharmacy, University of Bonn, Bonn, Germany ${ }^{2}$ Department of Internal Medicine, Johanniter Hospital, Bonn, Germany ${ }^{3}$ Oncology Practice, Bonn, Germany

${ }^{4}$ Specialist Breast Unit/ Senology, St Elisabeth Hospital Cologne-Hohenlind, Cologne, Germany

${ }^{5}$ Department of Informatics and Epidemiology, Institute for Medical Biometry, University of Bonn, Bonn, Germany

\section{Correspondence to} Dr Ulrich Jaehde; u.jaehde@uni-bonn.de

\section{ABSTRACT}

Objective: To develop and evaluate a multiprofessional modular medication management to assure adherence to capecitabine.

Methods: The study was conducted as a prospective, multicentred observational cohort study. All

participants received pharmaceutical care consisting of oral and written information. Daily adherence was defined as percentage of days with correctly administered capecitabine doses and assessed using medication event monitoring. According to their daily adherence during the first cycle, patients were identified as initially non-adherent ( $<90 \%$ adherence) or adherent ( $\geq 90 \%$ adherence). Initially non-adherent patients received additional adherence support.

Results: Seventy-three patients with various tumour entities were enrolled, 58 were initially adherent and 15 non-adherent. Median daily adherence of initially non-adherent patients increased from $85.7 \%$ to $97.6 \%$ during the observation period of six cycles.

Throughout all cycles, median daily adherence of initially adherent patients was $100.0 \%$. Daily adherence was not associated with sociodemographic and disease-related factors. No patient was non-persistent. Conclusions: An early adherence screening effectively distinguishes between patients adhering and nonadhering to capecitabine. The provision of specific adherence support is associated with enhanced adherence of initially non-adherent patients, whereas initially adherent patients remain adherent for at least six cycles without specific support. Our needs-based approach helps to use available resources for adherence management efficiently.

\section{INTRODUCTION}

Cancer therapy has traditionally been dominated by intravenously administered agents. ${ }^{1}$ However, oral anticancer drugs are increasingly used and more than one-quarter of all anticancer drugs currently under development are orally administered. ${ }^{2}{ }^{3}$ Oral anticancer therapies are highly accepted by patients based on obvious advantages, for example, higher convenience, avoidance of

\section{ARTICLE SUMMARY}

Article focus

- Adequate patient adherence to capecitabine, an orally administered prodrug of fluorouracil, is essential for treatment success. The early identification of potential non-adherers followed by adherence-enhancing measures may contribute to the effectiveness of oral anticancer drug therapy.

- This prospective, multicentred observational cohort study aimed to develop and evaluate a multiprofessional medication management to assure adherence to capecitabine.

- It was hypothesised that adherence of initially adherent patients $(\geq 90 \%$ adherence during the first cycle) would remain high over time without specific support and that initially non-adherent patients ( $<90 \%$ adherence during the first cycle) would benefit from specific adherence support.

Key messages

- An early adherence screening effectively distinguishes between patients adhering and nonadhering to capecitabine.

- The provision of specific adherence support is associated with enhanced adherence of initially non-adherent patients.

- Initially adherent patients remain adherent for at least six cycles without specific support implying that targeted support to those patients who benefit from it is a reasonable approach.

Strengths and limitations of this study

- Our approach is multiprofessional and needsbased utilising available resources for adherence management most efficiently.

- The relatively small sample size of initially nonadherent patients limits the validity of the observed results for this subgroup of patients.

venipuncture and paravasates as well as greater patient autonomy. ${ }^{2} 4{ }^{5}$ However, these treatments are also associated with many challenges. Owing to less intense contact between patient and healthcare providers, responsibilities in terms of managing the course of treatment are transferred to 
the patient such as monitoring of doses and toxicity. ${ }^{2} 6$ In contrast to intravenously administered anticancer treatments, healthcare providers cannot always assume that patients are adherent which is, however, the key prerequisite for treatment success. Multidisciplinary patient care and specific patient education regarding all aspects of the treatment regimen are crucial to maintain adherence. ${ }^{6-9}$

Patients of the present study were treated with the chemotherapeutic agent capecitabine, an orally administered prodrug of cytotoxic fluorouracil ( $5 \mathrm{FU}$ ). Capecitabine has an improved tolerability and comparable efficacy compared with infusional or bolus $5 \mathrm{FU}^{10}$ and is frequently used in the treatment of breast, colorectal and gastric cancer. Moreover, ovarian, pancreatic or oesophageal tumours may be treated with capecitabine. One capecitabine cycle consists of 3 weeks, 2 weeks of twice daily drug intake followed by 7 days of break. ${ }^{11}$

Patient adherence to prescribed treatment regimens for chronic non-oncologic diseases accounts for $50 \%$ on average only. ${ }^{12}{ }^{13}$ Medication-taking behaviour of patients with cancer is presumed to be particularly adherent, since cancer is a life-threatening disease. ${ }^{14-18}$ However, adherence rates of oral anticancer agents were reported to range from $16 \%$ to $100 \%$ depending on the drug and method of measurement. ${ }^{15}$ Exact measurement of adherence is a challenge and existing methods are limited for various reasons. ${ }^{19}$ Best estimation of adherence may be provided by electronic monitoring such as the medication event monitoring system (MEMS, Aardex Group Ltd., Zug, Switzerland) ${ }^{20}$

Several studies have been published investigating patient adherence to capecitabine. Partridge et al used MEMS for adherence assessment in older women with early-stage breast cancer. Adherence was defined as the number of doses taken divided by the doses expected. Seventy-five per cent of the included patients were regarded as adherent, that is, they performed more than $80 \%$ of the expected openings. Mean adherence accounted for $78 \%$ across all cycles. ${ }^{21}{ }^{22}$ Winterhalder et al used participant self-reports to explore adherence in patients with gastrointestinal and breast cancer. Any violation of the recommended treatment regimen, according to their diary entries, during the duration of the capecitabine treatment was considered as nonadherence. Ninety-one per cent $(161 / 177)$ patients were found to be fully adherent, whereas only $9 \%(16 / 177)$ reported some kind of adherence error. ${ }^{14}$ The adherence of 13 younger patients with metastatic breast cancer was assessed using MEMS and the median accounted for $96 \%$. Adherence was defined as observed doses divided by expected doses. Self-reported adherence was assessed additionally and the median was $97 \%$ $(\mathrm{n}=12){ }^{23}$ Self-reported non-adherence of 43 patients with breast and colorectal cancer was $23.3 \% .^{24}$ Furthermore, the effect of an intensified multidisciplinary pharmaceutical care programme consisting of a combination of written and spoken information on the adherence of patients with cancer taking capecitabine was investigated. Adherence was measured using MEMS and defined as the percentage of days with correct medication taking behaviour. Patients who received pharmaceutical care showed a significantly higher mean daily adherence compared with the control group who received standard care $(96.8 \%$ vs $87.2 \%, \mathrm{p}=0.029) .{ }^{25}$

Thus, adherence rates of patients treated with capecitabine are relatively high compared with non-oncologic oral drugs but can still be increased by specific measures. ${ }^{25}$ Conversely, this implies that only some patients treated with capecitabine are in need of an adherence-enhancing intervention and the limited resources could be used more efficiently. Certain patients manage their oral treatment regimen independently and do not benefit from specialised patient care. Therefore, we chose a modular medication management approach in this study. Patients with cancer were screened for their adherence during their first capecitabine cycle to detect potential nonadherers. Initially adherent as well as non-adherent patients received basic pharmaceutical care and adverse event management. Specific adherence support was only applied to initially non-adherent patients.

According to the recently published taxonomy for describing and defining adherence to medications ${ }^{26}$ this study primarily addressed the implementation element of adherence. The aim was to identify initially nonadherent patients and to investigate initially nonadherent and initially adherent patients' adherence over time. It was hypothesised that adherence of initially adherent patients would remain high over time without specific support and that initially non-adherent patients would benefit from specific adherence support.

\section{METHODS}

\section{Study design}

The study was conducted as a prospective, multicentred, two-arm observational cohort study. One study arm consisted of patients classified as initially adherent (baseline daily adherence $\geq 90 \%$ ), the other arm of initially nonadherent patients (baseline daily adherence $<90 \%$ ).

\section{Study setting and sample}

The study was conducted in two oncology outpatient wards and one oncology practice. Data were collected between July 2009 and March 2012. After the identification of eligibility by the collaborating oncologists, the study pharmacist asked the patients if they were willing to participate in the study. In case of acceptance, each participant signed a written informed consent. The study protocol considered a maximum observation period of six capecitabine cycles for every participant. The main inclusion criterion was the initiation of chemotherapy with capecitabine as a single agent or combination therapy for treatment of cancer. Patients had to be capecitabine-naïve, at least 18 years old and able to speak, read and write German. Inclusion had to 
take place within 2 weeks after initiation of capecitabine treatment. Exclusion criteria implied any diagnosis of a disease or mental state compromising full understanding of purpose and course of the study. The ethics committee of the University of Bonn, Germany voted positively for this study.

\section{Adherence measurement}

Adherence to capecitabine treatment was assessed using the MEMS. $^{27}$ Every participant was provided with a MEMS container and asked to use it for storage of capecitabine medication during study participation. For ethical reasons patients were informed about the fact that their adherence was being monitored. The caps of the MEMS containers recorded date and time of every opening. Patients were instructed to open the containers only when taking their capecitabine dose. In case of required refills, patients were requested to schedule refill and regular capecitabine intake at the same time in order to avoid additional openings. If this was not possible or in case of further extraordinary openings, patients were asked to note the respective information on a special documentation sheet. Since uncensored MEMS data might overestimate non-adherence ${ }^{28}$ adherence data were censored according to information derived from notes and interviews (eg, exclusion of self-reported nonmonitoring intervals or extra openings, and intake of doses taken from another source than MEMS). Measurement ended after six completed capecitabine cycles or in case of premature treatment discontinuation.

\section{Adherence analysis}

Adherence was studied using medication taking profiles uploaded from the MEMS monitors and patients' information concerning extraordinary incidents. 'Daily adherence' was selected as primary endpoint. It was defined as percentage of days with correctly administered capecitabine doses (number of days with correct drug intake divided by number of observed days). In the case of missing MEMS data the corresponding days were not included in the analysis, that is, the number of observed days was reduced accordingly. Adherence was assessed on days with drug intake as well as days during the rest period. A day was considered as adherent only, if two openings of the MEMS monitor were recorded on a day during the drug-intake period (dosing interval $\geq 6 \mathrm{~h}$ ) or if no openings were recorded during the rest period.

Different measures of adherence were used. 'Daily adherence' was calculated for every individual cycle on the basis of days with and without drug intake. Furthermore, 'daily intake adherence' was calculated for every individual cycle on the basis of the drug-intake interval only. This was performed in order to exclude the influence of the intake-free interval on the adherence. Additionally, 'persistence' of drug intake was analysed. Duration of physician's capecitabine prescription was compared with the duration of the actual treatment by the participant.
For the classification of a participant as initially adherent or non-adherent, daily adherence was calculated for the intake period of the first cycle plus first day of the therapy-free interval. This parameter is referred to as 'baseline daily adherence'. A participant was classified as initially adherent (baseline daily adherence $\geq 90 \%$ ) or initially non-adherent (baseline daily adherence $<90 \%$ ). Since no consensual standard for the definition of sufficient adherence exists ${ }^{16}$ the threshold of $90 \%$ was defined empirically based on the results of an earlier research project. ${ }^{25}$ If assessment of baseline adherence resulted in a participant being initially non-adherent, adherence support was provided before the start of the second intake period.

\section{Modular medication management}

In addition to standard care provided by physicians and nurses of the respective study centre, medication management consisted of three modules. A detailed literature search was conducted to identify most valuable components of pharmaceutical care and adherence enhancement. On the basis of the reviewed literature the modules were developed, discussed and adapted. Every study participant received module 1 (basic pharmaceutical care) as well as module 2 (adverse event management). These modules were provided by a registered pharmacist of the Department of Clinical Pharmacy at the University of Bonn, Germany, in collaboration with the attending physicians and nurses. If a participant was initially nonadherent, the pharmacist delivered module 3 (adherence support) to the patient additionally.

Modules 1 and 2 were initiated after inclusion. Module 1 implied detailed medication history taking to perform drug-drug interaction checks and compile an individual medication plan. In case of identified drug-related problems, necessary changes of the medication were made in collaboration with the responsible physician. Patients were educated in detail about the cytotoxic agent capecitabine, its mechanism of action and the individual dosing regimen. Further anticancer agents, supportive therapy and other agents taken regularly were also addressed. Patient counselling was supported by the provision of written information material. Within module 2, patients were educated regarding common adverse effects (eg, hand-foot syndrome and diarrhoea). Prophylaxis, detection and treatment of adverse effects were discussed in detail. If patients took other drugs or were prescribed a concomitant anticancer treatment, they were counselled regarding the adverse effects of these drugs as well. An information brochure regarding prevention and management of adverse effects caused by chemotherapy supported oral counselling.

Since feeding back of electronically compiled adherence data to the patients has been demonstrated to be an effective approach to enhance adherence, ${ }^{29}$ module 3 contained a detailed discussion of the patient's individual adherence results on the basis of cycle 1 MEMS data. Adherence support focused on the identification of 
reasons for non-adherence to define a feasible adherence-enhancing strategy. Since various types of nonadherence exist, strategies to overcome individual barriers to adherence were designed individually. Strategies to improve unintentional non-adherence (eg, due to forgetfulness) included treatment diaries or linking drug intake with a certain act of daily routine (cue dosing). In contrast, intentional non-adherence had to be approached in a completely different manner. If an adverse effect was the reason for not taking capecitabine, management and prevention of further adverse effects were addressed in accordance with module 2. Patients' expectations and experiences were included in all considerations. Moreover, an increase of the patient's awareness of the importance of adherence with capecitabine treatment was aimed. Routinely, beginning and end of the current and next capecitabine cycle were explicitly discussed. The content and course of the adherence-supporting session was adapted according to the patients' medication taking behaviour. If the participant showed a daily adherence $<90 \%$, the content of the first counselling session of module 3 was repeated and adherence-enhancing strategies were reassessed, discussed and adapted.

Personal follow-up visits took place at least once every cycle. Between scheduled appointments every participant had the possibility to reach individual advice in person, by telephone or by email.

\section{Sample size calculation and statistical analysis}

Sample size calculations were based on one-sided exact binomial tests and conducted for the primary endpoint 'daily adherence'. Available adherence data ${ }^{25}$ were analysed with regard to daily adherence of the participant's first capecitabine cycle. Regarding initially adherent patients a sample size of 45 was required to show with a power $(1-\beta)$ of $80 \%$ that $>75 \%$ of these patients remain being adherent (type I error $(\alpha)=5 \%$ ). The true population value of patients who persist being adherent was assumed to account for $>90 \%$. Regarding initially nonadherent patients, a sample size of 30 patients was required to show with a power $(1-\beta)$ of $80 \%$ that $>80 \%$ of these patients become adherent after the adherence support (type I error $(\alpha)=5 \%$ ). The true population value of patients who became adherent was assumed to account for $>95 \%$. Finally a dropout rate of $20 \%$ was estimated so that a total sample size of 90 patients resulted (54 initially adherent and 36 initially non-adherent patients). Data entry and statistical data analysis were carried out using Excel 2007 (Microsoft, Redmond, Washington, USA) and SPSS V.20 (SPSS Inc., Chicago, Illinois, USA, Statistical Package for the Social Sciences). Appropriate descriptive statistics was used to characterise the patient population and summarise the study results. Data were mostly binary, nominal, ordinal or failed to follow a normal distribution, thus non-parametric testing was utilised consistently. Differences regarding sociodemographic and disease-related characteristics between initially adherent and non-adherent patients were tested using the Fisher's exact test for nominal data. To explore the relationship between adherence and potential predictors of adherence, Spearman's rank correlation coefficient was used for comparing two continuous data sets and Mann-Whitney $U$ analysis was used for comparing continuous (not normally distributed) data with binary data sets.

\section{RESULTS}

During the data collection period participating oncologists assessed in total 97 patients for eligibility, 78 were enrolled in the study. Figure 1 provides a detailed overview of patient recruitment including reasons for
Figure 1 Patient recruitment flow diagram.
First patient in 07/2009, last patient in $11 / 2011$, last patient out $03 / 2012$

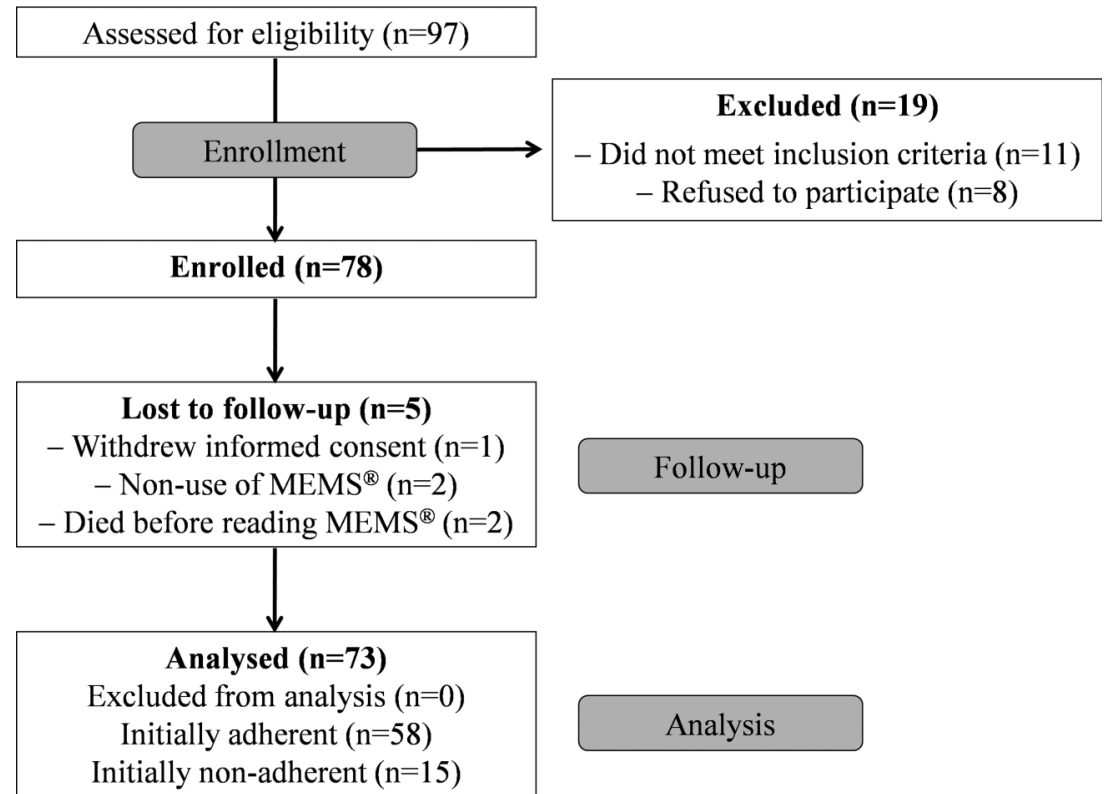


exclusion and loss to follow-up. The main reason (seven of eight refusals) for non-participation was perceived stress by the study in addition to their mentally and/or physically impaired condition. Since five patients were not capecitabine-naive, two patients were not able to speak, read and write German and for four patients MEMS use was not possible due to participation in another trial, they were not enrolled.

\section{Patient characteristics}

Seventy-three patients were analysed for baseline daily adherence, $58(79.5 \%)$ were initially adherent and 15 $(20.5 \%)$ initially non-adherent. Table 1 shows that there was no statistically significant difference between initially adherent and non-adherent patients regarding sociodemographic and disease-related characteristics. However, there was a significant difference in the therapy setting ( $p=0.021$, Fisher's exact test $)$.

\section{Initially adherent patients}

Initially adherent patients were observed for a median time of 119.0 days (range 21.0-152.0; IQR=69.8-126.0). During all observed cycles, a high percentage of these patients showed a daily adherence $\geq 90 \%$ (figure $2 \mathrm{~A}$ ). After the sixth cycle, 36 of $37(97.3 \%$, CI $88.8 \%$ to 99.4\%) initially adherent patients showed a daily adherence $\geq 90 \%$. Since the CI does not include $75 \%$ it is shown with a type I error of $5 \%$ that more than $75 \%$ of the initially adherent patients remained adherent after the modular medication management (without specific adherence support).

Figure 2B shows the same kind of data analysis for the daily intake adherence (excluding therapy-free interval).

Table 1 Sociodemographic and disease-related patient characteristics

\begin{tabular}{|c|c|c|c|c|c|}
\hline \multirow[b]{2}{*}{ Sociodemographic characteristics } & \multicolumn{2}{|c|}{$\begin{array}{l}\text { Initially } \\
\text { adherent }\end{array}$} & \multicolumn{2}{|c|}{$\begin{array}{l}\text { Initially } \\
\text { non-adherent }\end{array}$} & \multirow[b]{2}{*}{ p Value } \\
\hline & $\overline{\mathrm{n}}$ & $\%$ & $\overline{\mathbf{n}}$ & $\%$ & \\
\hline \multicolumn{6}{|l|}{ Classified age (years) } \\
\hline$\leq 50$ & 11 & 19.0 & 0 & 0.0 & \multirow[t]{5}{*}{0.203} \\
\hline$\overline{5} 1-60$ & 15 & 25.9 & 6 & 40.0 & \\
\hline $61-70$ & 17 & 29.3 & 3 & 20.0 & \\
\hline $71-80$ & 10 & 17.2 & 5 & 33.3 & \\
\hline$>80$ & 5 & 8.6 & 1 & 6.7 & \\
\hline \multicolumn{6}{|l|}{ Sex } \\
\hline Female & 44 & 75.9 & 10 & 66.7 & \multirow[t]{2}{*}{0.516} \\
\hline Male & 14 & 24.1 & 5 & 33.3 & \\
\hline \multicolumn{6}{|c|}{ Number of additional drugs (excluding PRN drugs) } \\
\hline$\leq 5$ & 45 & 77.6 & 10 & 66.7 & \multirow[t]{4}{*}{0.514} \\
\hline $6-10$ & 9 & 15.5 & 3 & 20.0 & \\
\hline$>10$ & 3 & 5.2 & 2 & 13.3 & \\
\hline No answer & 1 & 1.7 & 0 & 0.0 & \\
\hline \multicolumn{6}{|l|}{ Tumour entity } \\
\hline Breast cancer & 21 & 36.2 & 7 & 46.7 & \multirow[t]{8}{*}{0.818} \\
\hline Colorectal cancer & 25 & 43.1 & 7 & 46.7 & \\
\hline Gastric cancer & 3 & 5.2 & 0 & 0.0 & \\
\hline Oesophageal cancer & 1 & 1.7 & 1 & 6.7 & \\
\hline Ovarian cancer & 3 & 5.2 & 0 & 0.0 & \\
\hline Cancer of unknown primary & 1 & 1.7 & 0 & 0.0 & \\
\hline Pancreatic cancer & 3 & 5.2 & 0 & 0.0 & \\
\hline Endometrial cancer & 1 & 1.7 & 0 & 0.0 & \\
\hline \multicolumn{6}{|l|}{ Therapy regimen at inclusion } \\
\hline Monotherapy & 35 & 60.3 & 7 & 46.7 & \multirow[t]{2}{*}{0.339} \\
\hline Combination therapy & 23 & 39.7 & 8 & 53.3 & \\
\hline \multicolumn{6}{|l|}{ Treatment intention } \\
\hline Curative & 8 & 13.8 & 3 & 20.0 & \multirow[t]{2}{*}{0.686} \\
\hline Palliative & 50 & 86.2 & 12 & 80.0 & \\
\hline \multicolumn{6}{|l|}{ Classified time since diagnosis } \\
\hline$<1 / 2$ year & 15 & 25.9 & 4 & 26.7 & \multirow[t]{3}{*}{0.712} \\
\hline $1 / 2$ to 2 years & 22 & 37.9 & 4 & 26.7 & \\
\hline$>2$ years & 21 & 36.2 & 7 & 46.7 & \\
\hline \multicolumn{6}{|l|}{ Therapy setting } \\
\hline Oncology outpatient ward & 51 & 87.9 & 9 & 60.0 & \multirow[t]{2}{*}{0.021} \\
\hline Oncology practice & 7 & 12.1 & 6 & 40.0 & \\
\hline
\end{tabular}




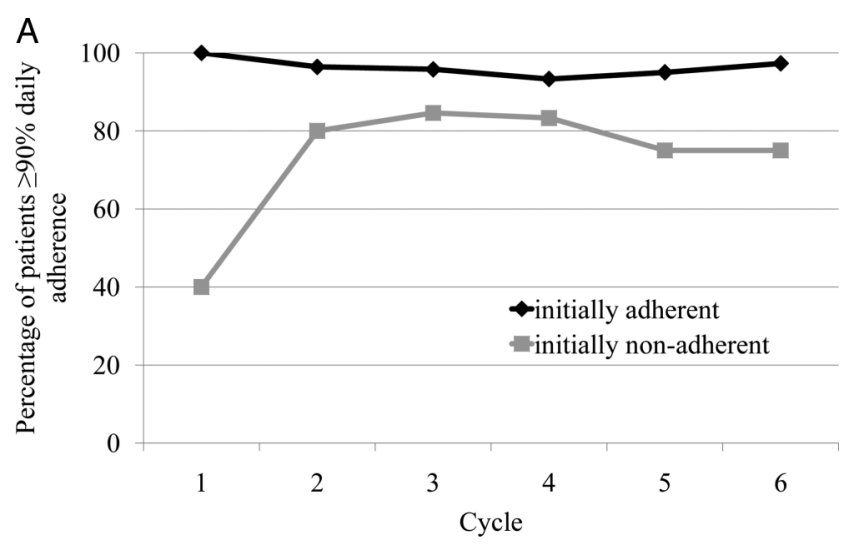

$\mathrm{B}$

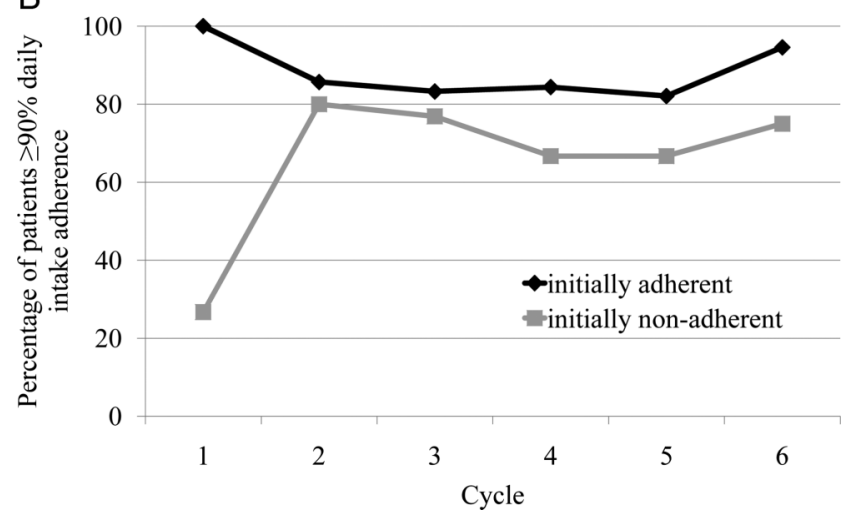

Figure 2 Percentage of patients exhibiting a (A) daily adherence $\geq 90 \%$ (during intake and rest periods) and a (B) daily intake adherence $\geq 90 \%$ (during the intake periods only).

The fraction of initially adherent patients with a daily intake adherence $\geq 90 \%$ was lower compared with daily adherence reflecting that adherence is lower during intake than rest periods.

Figure 3 demonstrates that variability with regard to daily adherence increased from cycle 1 compared with further cycles. Median daily adherence was $100 \%$ in every cycle. Mean daily adherence decreased from $98.9 \%$ in cycle 1 to $97.3 \%$ in cycle 6 . Online supplementary table A provides more detailed information. Although initially adherent patients did not receive specific adherence support, a consistently high median daily adherence in a majority of these patients was observed. Only in exceptional cases median daily adherence was observed to be lower than $90 \%$. Individual daily adherence profiles of each patient over the observation period are provided in online supplementary figure A.

\section{Initially non-adherent patients}

Initially non-adherent patients were observed for a median time of 118.0 days (range 35.0-140.0; IQR=96.0126.0). Figure 2A illustrates the percentage of patients who showed a daily adherence $\geq 90 \%$ during the different cycles. Adherence increased in association with the specific support provided. In cycle 2 the percentage of adherent patients was $80.0 \%(12 / 15)$ compared with

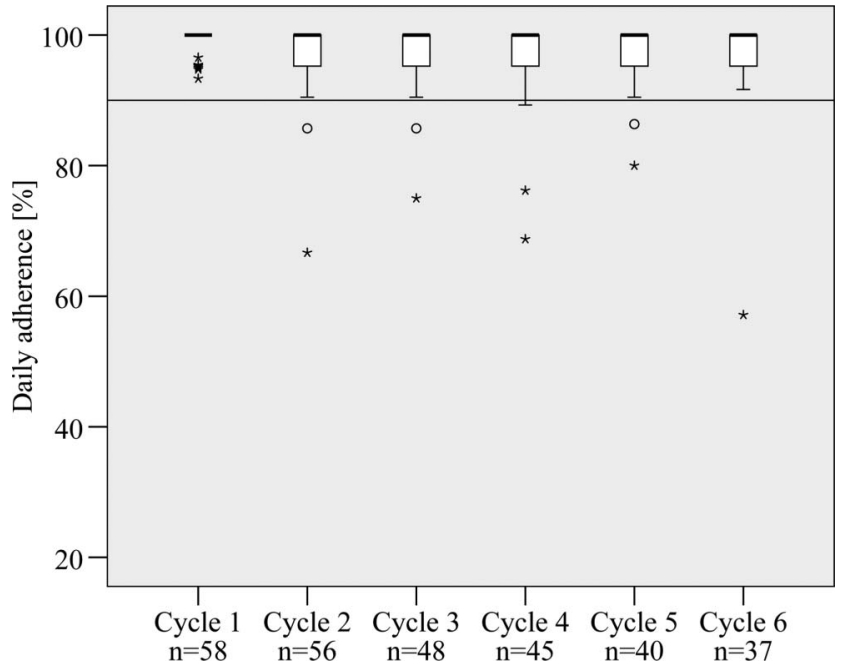

Figure 3 Daily adherence of initially adherent patients during cycle 1-6 (the median is represented by the black band in every box; bottom and top of each box are the first and third quartiles; circles are 1.5-3 times the box height away from the box; stars are $>3$ times the box height away from the box).

$40.0 \%(6 / 15)$ in cycle 1 and it ranged between $75.0 \%$ and $84.6 \%$ in the following cycles 3-6. After completion of the sixth cycle, daily adherence of six of eight $(75.0 \%$, CI $46.0 \%$ to $91.3 \%)$ initially non-adherent patients accounted for $\geq 90 \%$. Since the CI included $80 \%$ which was the cut-off value used for sample size determination of initially non-adherent patients, it could not be proven that $>80 \%$ of initially non-adherent patients were adherent after the intervention.

Figure 2B shows the percentage of initially nonadherent patients with a daily intake adherence $\geq 90 \%$ over the cycles. In contrast to the initially adherent patients, the fractions of initially non-adherent patients exhibiting a daily adherence $\geq 90 \%$ and a daily intake adherence $\geq 90 \%$ did not exhibit major differences.

Median daily adherence increased from $85.7 \%$ in cycle 1 to $97.6 \%$ in cycle 6 (see figure 4). Mean daily adherence accounted for $80.8 \%$ during the first cycle and was found to be greater than $90 \%$ during the application of the adherence support module (see online supplementary table B). Adherence varied widely between patients but also from cycle to cycle in the same patients. Online supplementary figure B shows individual daily adherence profiles of initially nonadherent patients during the course of the study calculated for intake plus rest period.

\section{Potential predictors of adherence}

There was no indication of an existing relationship between patients' daily adherence during the first cycle and their age (Spearman's $\mathrm{r}=0.009, \mathrm{p}=0.941$ ) or gender ( $\mathrm{p}=0.891$, Mann-Whitney- $U$ test). In addition, there was not any significant association between daily adherence and any further sociodemographic and disease-related characteristics. 


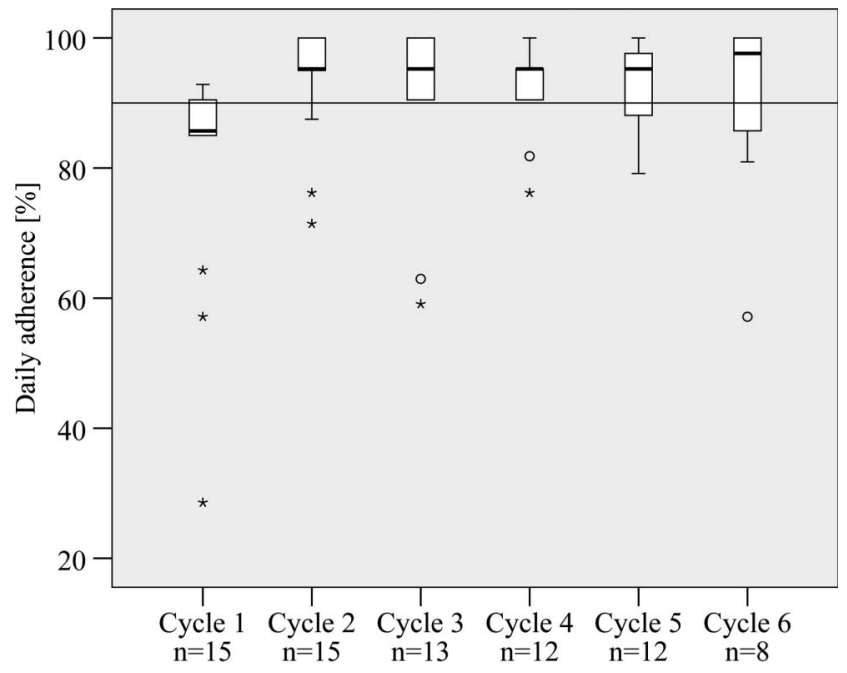

Figure 4 Daily adherence of initially non-adherent patients during cycle 1-6 (the median is represented by the black band in every box; bottom and top of each box are the first and third quartiles; circles are 1.5-3 times the box height away from the box; stars are $>3$ times the box height away from the box).

\section{Persistence}

All study patients were persistent during the whole period they were prescribed capecitabine chemotherapy. No patient performed an unauthorised discontinuation of his capecitabine treatment.

However, in 17 of the $58(29.3 \%)$ initially adherent patients capecitabine therapy was discontinued prematurely by the physicians. In 12 patients this decision was taken due to tumour progression. Further reasons for therapy discontinuation were adverse drug reactions (hand-foot syndrome and haemolytic anaemia), hospital admission, the toxicity of a coadministered drug and the patient's wish to stop treatment. Thirty-six (62.1\%) patients completed six cycles as planned, two patients $(3.4 \%)$ completed less than six capecitabine cycles as planned, one patient $(1.7 \%)$ died after the completion of the third cycle and two patients quit their study participation during the second cycle.

In 5 of $15(33.3 \%)$ initially non-adherent patients capecitabine therapy was discontinued prematurely due to tumour progression. Eight patients $(53.3 \%)$ completed six capecitabine cycles as planned, one patient $(6.7 \%)$ completed five cycles as planned and one patient died during the second cycle.

\section{DISCUSSION}

In this study, we applied a systematic screening for nonadherent patients at an early stage of their capecitabine chemotherapy to provide a patient-tailored modular medication management. The results indicate that specific adherence support might improve adherence of initially non-adherent patients to capecitabine and that initially adherent patients' medication taking behaviour persists over time under basic pharmaceutical care and adverse event management.

\section{Sample size of initially non-adherent patients}

A major limitation of our study is the relatively small number of initially non-adherent patients. Instead of the required sample size of 30 initially non-adherent patients, only 15 patients could be enrolled during the study period. Previous data suggested a distribution of $60 \%$ initially adherent and $40 \%$ initially non-adherent patients. ${ }^{25}$ The actual distribution within our patient population was 80-20\%. This has to be considered before interpreting data of the initially non-adherent patients. However, a clear trend towards an improved adherence over time was observed. Further multicentre studies are needed to provide better generalisable findings.

\section{Adherence screening}

For the classification of patients as initially non-adherent or adherent, we used daily adherence of the first drug-intake period plus the first day of the therapy-free interval assessed by MEMS. Consideration of the whole capecitabine cycle would have provided a more complete picture of the participant's adherence during the first cycle. However, this was not feasible. To initiate adherence support before the start of cycle 2, an exact appointment on day 21 of the first cycle for group allocation would have been necessary. A belated start of the adherence supporting module would have biased the results of initially non-adherent patients.

Our approach using the gold standard of adherence assessment was suitable to discriminate between adhering and non-adhering patients. In theory it would be less costly and labour intensive to identify non-adhering patients alternatively by means of possible predictors, for example, by a specific questionnaire. In general, numerous factors associated with non-adherence to oral anticancer drugs have been identified like, for example, side effects, forgetfulness or disliking aspects of treatment. ${ }^{20}{ }^{30}$ On the basis of our data, it was, however, not possible to derive significant information on adherence from sociodemographic or disease-related characteristics, for example, age. Indeed, we observed that the three patients exhibiting the lowest baseline adherence during cycle $1(28.6 \%, 57.1 \%$ and $64.3 \%)$ were of a relatively old age (90, 75 and 79 years). However, from this result it cannot be concluded that adherence decreases with increasing age as there were also elderly patients exhibiting high adherence. Our findings are in line with the findings of Partridge $e t a l^{21}$ who did not find an association of adherence and age. Furthermore, Bhattacharya et al did not identify significant associations between self-reported adherence to capecitabine and experience of side effects, beliefs about capecitabine or satisfaction with information. However, the generalisability of that study was also limited by a relatively small sample size. ${ }^{24}$ Therefore, larger multicentre studies are 
necessary to identify precise predictors of nonadherence to capecitabine.

\section{Effect of modular medication management}

Adherence rates in our study were higher than those reported by Partridge $e t a l^{21}$ who found an average overall adherence measured by MEMS (defined as the number of doses taken divided by the number of doses prescribed) between $70 \%$ and $80 \%$. Analysing our data the same way, overall adherence values ranged between $98.2 \%$ and $100.5 \%$ in initially adherent patients and between $93.8 \%$ and $102.7 \%$ in initially non-adherent patients. High adherence results in this study might be explained by the fact that every participant of the present study received two pharmaceutical care modules during all six cycles. Regardless the specific adherence support, elements of module 1 and 2, such as an individual medication plan and patient counselling regarding prophylaxis, detection and treatment of adverse effects, might have had a beneficial effect on adherence of initially adherent as well as initially non-adherent patients as shown previously. ${ }^{25}$

However, in case of initially non-adherent patients, the provided adherence support might have increased adherence additionally. This finding is consistent with previous results from our working group. Under the provision of intensified pharmaceutical care to 48 patients with breast and colorectal cancer, the intervention group showed an increased mean overall adherence in comparison with the control group. ${ }^{25}$ In line with previous results $^{21} 25$ non-persistence did not present a problem in our group of patients.

\section{Daily adherence versus daily intake adherence}

Daily adherence during the intake periods of each cycle was generally lower compared with daily adherence calculated on the basis of drug intake plus rest period. This implies that adherence to the regimen was better in the rest period when the drug should not be taken, that is, not many patients took the drug by mistake. However, 8 of $15(53.3 \%)$ patients took capecitabine 1 day too long, too short or completely ignored the break. From this finding we conclude that special attention has to be paid to the change of drug-intake days to drug-free days in the first capecitabine cycle. Patients have to be educated in detail regarding this particularity of the capecitabine treatment regimen. The attending healthcare provider should inform the patient exactly on the dates of the intake-free period. Written notes can serve as mnemonic devices. Future studies should facilitate the development of appropriate adherence parameters in order to improve the reflection of the longitudinal aspect of adherence data.

\section{Adherence management}

Even though daily adherence could be improved in initially non-adherent patients, it has to be pointed out that this patient population did not reach the same adherence level as initially adherent patients. Moreover, interindividual variability of adherence was higher. This finding suggests that a subgroup of patients with low adherence benefits from the adherence-enhancing intervention as suggested by Simons $e t a l^{25}$ However, a certain number of patients cannot be reached and reveals a resistant medication taking behaviour. Reasons for intentional non-adherence in those patients were difficulties in swallowing tablets due to nausea and emesis caused by capecitabine (despite the provision of antiemetic prophylaxis and treatment), averseness to medication or 'compensating' intake for previous non-adherence during treatment break. Unintentional non-adherence was mainly based on forgetfulness. Further research should include a systematic approach to develop strategies for adherence management in those 'resistant' patients. The adherence of intentionally non-adherent patients could be enhanced by means of advanced educational interventions. Behavioural interventions such as medication dosette boxes or alarm clocks could be used more extensively in the adherence enhancement of unintentionally non-adherent patients.

\section{CONCLUSIONS}

In summary, the results of this study demonstrate the potential of an early adherence screening for non-adherence and an individually applied modular medication management to use limited resources most efficiently. The provided adherence support is associated with enhanced adherence of initially non-adherent patients to oral chemotherapy. Moreover, the provision of basic pharmaceutical care and adverse event management was sufficient to maintain adherence in initially adherent patients for at least six cycles. The identification of potential predictors of adherence would facilitate the utilisation and broad application of the proposed adherence screening and modular medication management.

Acknowledgements We thank all patients who were willing to participate in our study. Furthermore, we thank Regina Moka and Friederike Schroeder as well as all oncologists and nurses who supported the conduction of our study.

Contributors LK and UJ conceived the study design, substantially contributed to data analysis and interpretation, and drafted the manuscript. LK, YDK, PFS and CS were involved in the data collection and provision of multidisciplinary patient care. RF contributed substantially to statistical data analysis and interpretation. UJ is the guarantor. All authors critically reviewed the manuscript and gave their final approval for the version to be published.

Funding A supplementary grant was provided by Roche, Basel. However, the researchers were entirely independent during all phases of this work.

\section{Competing interests None.}

Patient consent Obtained.

Ethics approval This study was conducted with the approval of the ethics committee of the University of Bonn, Germany (consecutive number 042/09).

Provenance and peer review Not commissioned; externally peer reviewed.

Data sharing statement Extra data is available by e-mailing Ulrich Jaehde (u.jaehde@uni-bonn.de). All authors, external and internal, had full access to all of the data (including statistical reports and tables) in the study and can 
take responsibility for the integrity of the data and the accuracy of the data analysis.

Open Access This is an Open Access article distributed in accordance with the Creative Commons Attribution Non Commercial (CC BY-NC 3.0) license, which permits others to distribute, remix, adapt, build upon this work noncommercially, and license their derivative works on different terms, provided the original work is properly cited and the use is non-commercial. See: http:// creativecommons.org/licenses/by-nc/3.0/

\section{REFERENCES}

1. Findlay M, Minckwitz G Von, Wardley A. Effective oral chemotherapy for breast cancer: pillars of strength. Ann Oncol 2008;19:212-22.

2. Weingart SN, Brown E, Bach PB, et al. NCCN Task Force Report: oral chemotherapy. J Natl Compr Canc Netw 2008;6(Suppl 3): S1-14.

3. Halfdanarson TR, Jatoi A. Oral cancer chemotherapy: the critical interplay between patient education and patient safety. Curr Oncol Rep 2010;12:247-52.

4. Schott S, Schneeweiss A, Reinhardt J, et al. Acceptance of oral chemotherapy in breast cancer patients-a survey study. BMC Cancer 2011;11:129.

5. Górnaś M, Szczylik C. Oral treatment of metastatic breast cancer with capecitabine: what influences the decision-making process? Eur $J$ Cancer Care (Engl) 2010;19:131-6.

6. Weingart SN, Flug J, Brouillard D, et al. Oral chemotherapy safety practices at US cancer centres: questionnaire survey. BMJ 2007;334:407.

7. Wood L. A review on adherence management in patients on oral cancer therapies. Eur J Oncol Nurs 2012;16:432-8.

8. Parsad SD, Ratain MJ. Prescribing oral chemotherapy. BMJ 2007;334:376

9. Schneider SM, Hess K, Gosselin T. Interventions to promote adherence with oral agents. Semin Oncol Nurs 2011;27:133-41.

10. Cassidy J. First-line oral capecitabine therapy in metastatic colorectal cancer: a favorable safety profile compared with intravenous 5-fluorouracil/leucovorin. Ann Oncol 2002;13:566-75.

11. Walko CM, Lindley C. Capecitabine: a review. Clin Ther 2005;27:23-44.

12. Sabaté E. Adherence to long-term therapies: evidence for action Geneva: WHO, 2003

13. Cutler DM, Everett W. Thinking outside the pillbox-medication adherence as a priority for health care reform. $N$ Engl J Med 2010;362:1553-5.

14. Winterhalder R, Hoesli P, Delmore G, et al. Self-reported compliance with capecitabine: findings from a prospective cohort analysis. Oncology 2011;80:29-33.

15. Ruddy K, Mayer E, Partridge A. Patient adherence and persistence with oral anticancer treatment. CA Cancer J Clin 2009;59:56-66.
16. Osterberg L, Blaschke T. Adherence to medication. N Engl J Med 2005;353:487-97.

17. Waterhouse DM, Calzone KA, Mele C, et al. Adherence to ora tamoxifen: a comparison of patient self-report, pill counts, and microelectronic monitoring. J Clin Oncol 1993;11:1189-97.

18. Haynes RB, McDonald HP, Garg AX. Helping patients follow prescribed treatment: clinical applications. JAMA 2002;288:2880-3.

19. Spoelstra SL, Given CW. Assessment and measurement of adherence to oral antineoplastic agents. Semin Oncol Nurs 2011;27:116-32.

20. Foulon V, Schöffski P, Wolter P. Patient adherence to oral anticancer drugs: an emerging issue in modern oncology. Acta Clin Belg 2011;66:85-96.

21. Partridge $A H$, Archer L, Kornblith $A B$, et al. Adherence and persistence with oral adjuvant chemotherapy in older women with early-stage breast cancer in CALGB 49907: adherence companion study 60104. J Clin Oncol 2010;28:2418-22.

22. Muss HB, Berry DA, Cirrincione CT, et al. Adjuvant chemotherapy in older women with early-stage breast cancer. $N$ Engl J Med 2009;360:2055-65.

23. Mayer EL, Partridge $\mathrm{AH}$, Harris LN, et al. Tolerability of and adherence to combination oral therapy with gefitinib and capecitabine in metastatic breast cancer. Breast Cancer Res Treat 2009;117:615-23.

24. Bhattacharya D, Easthall C, Willoughby KA, et al. Capecitabine non-adherence: exploration of magnitude, nature and contributing factors. J Oncol Pharm Pract 2012;18:333-42.

25. Simons S, Ringsdorf S, Braun M, et al. Enhancing adherence to capecitabine chemotherapy by means of multidisciplinary pharmaceutical care. Support Care Cancer 2011;19:1009-18.

26. Vrijens B, Geest S De, Hughes DA, et al. A new taxonomy for describing and defining adherence to medications. Br J Clin Pharmacol 2012;73:691-705.

27. Cramer JA. Microelectronic systems for monitoring and enhancing patient compliance with medication regimens. Drugs 1995;49:321-7.

28. Denhaerynck K, Schäfer-Keller P, Young J, et al. Examining assumptions regarding valid electronic monitoring of medication therapy: development of a validation framework and its application on a European sample of kidney transplant patients. BMC Med Res Methodol 2008:8:5

29. Demonceau J, Ruppar T, Kristanto P, et al. Identification and assessment of adherence-enhancing interventions in studies assessing medication adherence through electronically compiled drug dosing histories: a systematic literature review and meta-analysis. Drugs 2013;73:545-62.

30. Noens L, Van Lierde M, Bock R De, et al. Prevalence, determinants, and outcomes of nonadherence to imatinib therapy in patients with chronic myeloid leukemia: the ADAGIO study. Blood 2009;113:5401-11. 